\title{
Early Neuromechanical Outcomes of the Triceps Surae Muscle-Tendon After an Achilles' Tendon Repair
}

\author{
Hsing-Kuo Wang, PT, MSc, MMedSci, PhD, ${ }^{a, b}$ Hongsen Chiang, MD, MSc, ${ }^{c}$ \\ Wen-Shiang Chen, MD, PhD, ${ }^{\text {, e }}$ Tiffany Tingfang Shih, MD, PhD, ${ }^{f, g}$ \\ Yung-Cheng Huang, MD, MSc, ${ }^{\text {h }}$ Ching-Chuan Jiang, MD, MSc ${ }^{c}$
}

From the ${ }^{a}$ School and Graduate Institute of Physical Therapy, College of Medicine, National Taiwan University, Taipei; ${ }^{b}$ Center of Physical

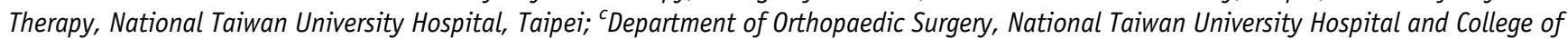
Medicine, National Taiwan University, Taipei; ${ }^{d}$ Department of Physical Medicine \& Rehabilitation, National Taiwan University Hospital, Taipei; ${ }^{e}$ Department of Physical Medicine \& Rehabilitation, College of Medicine, National Taiwan University, Taipei; ${ }^{f}$ Department of Medical Image, National Taiwan University Hospital, Taipei; ${ }^{g}$ Department of Surgery, College of Medicine, National Taiwan University, Taipei; and ${ }^{h}$ Department of Physical Medicine and Rehabilitation, Cheng Hsin General Hospital, Taipei, Taiwan, ROC.

\begin{abstract}
Objectives: To compare the neuromechanical and functional characteristics of the legs of athletes who underwent unilateral Achilles' tendon repair and their controls, and to determine any correlation between the characteristics.

Design: A case-control and cross-sectional study.

Setting: A university institute.

Participants: Male athletes $(\mathrm{N}=33$ ) were recruited; 23 in the $\geq 3$ - and $<12$-month postsurgical group (median age, 29.8y; age range, 21.9-40.0y) and 10 in the control group (median age, 30.0y; age range, 21.1-39.5y) who had not undergone any surgery.

Intervention: Surgical Achilles' tendon repair in the study group.

Main Outcome Measures: Bilateral measurements of activation strategy involving the triceps surae and tibialis anterior muscles, mechanical properties of the Achilles' tendon, and explosive performance tests were conducted.

Results: Compared with the noninjured legs and the control legs, the repaired legs showed lower normalized rates of electromyographic rise (RER) in the soleus, gastrocnemius medialis, and gastrocnemius lateralis ( $P$ ranged between .006 and .001$)$; and less tendon stiffness, greater hysteresis, and less rates of force development (RFD) ( $P$ ranged between .006 and <.001). Repaired legs had less ankle dorsiflexion, a shorter 1-leg hopping distance, and lower balance scores $(P \leq .001)$. The noninjured legs of the athletes who underwent surgical Achilles' tendon repair had a lower normalized RER $(0-50 \mathrm{~ms})$ in the soleus and lateral gastrocnemius when compared with the legs of the healthy controls $(P=.011)$. The neuromechanical outcomes and explosive performances showed correlations with RFD, 1-leg hopping distance, and balance score. Conclusions: The athletes who underwent unilateral Achilles' tendon repair demonstrated bilateral neuromechanical deficits within the 1-year postsurgical period.
\end{abstract}

Archives of Physical Medicine and Rehabilitation 2013;94:1590-8

(C) 2013 by the American Congress of Rehabilitation Medicine

\begin{abstract}
Achilles' tendon ruptures are common in ball games. ${ }^{1}$ Ruptures of tendinous tissue that connects the muscles under neural control lead to altered neural or mechanical transduction in the muscle-

Supported by the National Science Council, Republic of China (grant no. NSC98-2314-B-002013-MY3).

No commercial party having a direct financial interest in the results of the research supporting this article has or will confer a benefit on the authors or on any organization with which the authors are associated.
\end{abstract}

tendon unit. ${ }^{2,3}$ Within 1 year of Achilles' tendon repair surgery, young adults show altered soleus muscle activation and antagonist coactivation, as well as increased passive ankle stiffness ( $\Delta$ moment $/ \Delta$ degree of dorsiflexion angle) in their repaired legs during walking. ${ }^{2}$ Image studies have demonstrated that tendon stiffness was decreased within a follow-up period of 6 to 18 weeks to 1 year after Achilles' tendon repair in recreational athletes. The tendon module of elasticity (strain under defined loading/ 
transverse area) was correlated to the heel raise index; that is, the product of the number of heel raises and height is normalized as a percentage of the other side. ${ }^{3}$ The healing process after repairs seems to be regulated by a mechanism that tries to obtain optimal tendon stiffness. ${ }^{3}$ Collectively, this neuromechanical evidence in the triceps surae muscle-tendon unit within 1 year of Achilles' tendon repair indicates altered neural control and tissue mechanical properties in the region surrounding the ankle joints and suggests slow force production. ${ }^{4-6}$ Nonetheless, it was controversial to accept the noninjured legs as references for rehabilitation because these studies were performed without a healthy control group. We hoped to investigate the linkages between these postsurgical neuromechanical alterations and clinical performance deficits in order to improve the rehabilitation of injured athletes. To this end, we have focused on the neuromechanical profiles of the triceps surae muscle-tendon unit and functional assessments in the lower extremities within a period of 1 year after Achilles' tendon repair.

Electromyography, including rates of electromyographic rise (RER), is used to determine the ability for rapid activation of limb muscles. ${ }^{7}$ The RER is also a determinant for the rate of force development (RFD). ${ }^{7}$ Increases in absolute RFD imply a decrease in the time to develop a specific level of force output. In addition, RER ratios for the tibialis anterior and soleus muscles are used to indicate antagonist coactivation of the plantar flexor and dorsiflexor muscles during the early phase of explosive muscle contractions. ${ }^{6}$ The in vivo viscoelastic properties of the tendonaponeurosis complex including stiffness and mechanical hysteresis are often measured with real-time ultrasonography. ${ }^{8}$ Tendon stiffness is determined by tendon elongation over a given force range, and hysteresis refers to the ratio between dissipated and stored elastic energy under a stretch-recoil condition. ${ }^{9}$ Stiffness and mechanical hysteresis of tendons are relevant to mechanotransduction and movement efficiency. ${ }^{10}$ These parameters are associated with either the balance or fast force capacity that relates to skill fitness. ${ }^{7,11}$ A previous study ${ }^{6}$ has shown lower strengthindependent force development in plantar flexion and concomitantly higher RER ratios between the tibialis anterior and soleus in athletes with middle-portion Achilles' tendinopathy. These sophisticated techniques enable us to investigate profiles of neural control of leg muscles regarding the activation of the agonistantagonist muscles and tendon mechanical properties involving mechanotransduction and elastic energy utilization in an individual with tendon injuries. However, these techniques were not used to study subjects with an Achilles' tendon repair.

We hypothesize that within the first year of unilateral Achilles' tendon repair in athletes, there are significant differences in the neuromechanical outcomes and explosive performance in repaired legs when compared with contralateral noninjured legs and the legs of healthy control subjects. In addition, neuromechanical

\begin{tabular}{|rl|}
\hline List of abbreviations: \\
$\mathrm{M}_{\max }$ maximal M wave \\
$\mathrm{M}_{\text {sup }}$ & supramaximal $M$ wave \\
MVIC & maximal voluntary isometric contraction \\
RER & rate of electromyographic rise \\
RFD & rate of force development \\
RMS & root mean square \\
SEBT & Star Excursion Balance Test \\
VISA-A & Victorian Institute of Sports Assessment-Achilles \\
& questionnaire \\
\hline
\end{tabular}

outcomes correlate with explosive performance, including torque development, balance, or 1-leg jump length.

\section{Methods}

\section{Participants}

This study was approved by the institutional review board of National Taiwan University Hospital (reference no. 201102012RC). All participants provided written informed consent. Four surgical group practices specializing in foot and ankle disorders were invited to join the study, which took place from April 2011 to March 2012. The surgeons primarily used the Kessler suturing technique to repair ruptured Achilles' tendons and recommend a 16-week protocol after the repairs. ${ }^{12,13}$ This protocol was supervised and began with a toe motion/leg cast for the first 6 weeks, followed by a standardized weight-bearing, stretching, and strengthening regimen for 3 months. ${ }^{12,13}$ After the 16-week protocol, injured athletes are referred to a physical therapist depending on the demands of their sport and level. For this study, male athletes who had experienced unilateral, complete Achilles' tendon ruptures during sports activities within the year before study recruitment were included from outpatient clinics of these surgeons by the researchers. Male athletes were recruited because female hormones influence tendon-aponeurosis strain. ${ }^{14}$ The control subjects were matched to the physical characteristics of the study athletes, but had no history of Achilles' tendon pain or injury causing them to seek medical treatment or surgery; they were recruited from the same sports leagues. Sixteen potential control subjects and 28 subjects with unilateral Achilles' tendon repairs met the inclusion criteria and were recruited.

Before measurements were taken, injury histories, pain questionnaires, physical examinations, ${ }^{15}$ and ultrasonographic screenings ${ }^{16,17}$ with a 5- to $12-\mathrm{MHz}$ broadband linear array transducer ${ }^{\mathrm{a}}$ were obtained to ensure that subjects met the inclusion criteria and that there was no evidence of Achilles' tendinopathy in the noninjured legs or legs of the controls. The subjects with repaired Achilles' tendons indicated the clinical severity of their Achilles' tendon pain by filling out the Victorian Institute of Sports Assessment-Achilles (VISA-A) questionnaire. ${ }^{18}$ Assistance was provided, as needed, to translate the English content of the questionnaire to Mandarin Chinese. Subjects with repairs were excluded if they (1) had the repairs less than 3 months before recruitment; (2) were unable to partake in the measurements because of a subjective fear of rerupture ${ }^{19}$; (3) had delayed surgery ( $>1$ wk after injury) or were diagnosed with a sural nerve injury ${ }^{2}$; (4) did not complete the supervised 16-week protocol with a physiotherapist or trainer; or (5) exhibited any positive signs on physical examinations using the techniques of the Royal London Hospital test, ${ }^{15}$ or had ultrasonographic evidence indicating intratendinous local hypoechoic swelling or neovascularization ${ }^{20}$ in their noninjured contralateral leg. The exclusion criteria for the healthy control subjects were included in the fifth criterion listed for the subjects with repairs. Five potential subjects with unilateral Achilles' tendon repairs were excluded, 3 because of ultrasonographic evidence of tendinopathy in their noninjured control leg and 2 because of delayed surgery. Physical characteristics and VISA-A scores of the 23 subjects with repaired Achilles' tendons (6 badminton players, 3 football players, 3 basketball players, 3 sprinters, 3 volleyball players, 2 tennis players, 2 gymnasts, 1 long jumper) are summarized in table 1 . 
Table 1 Subjects' demographics and clinical severity after Achilles' tendon repairs

\begin{tabular}{lccc}
\hline Variable & Subjects With Achilles' Tendon Repair & Healthy Control Subjects & Mann-Whitney U Test $(P)$ \\
\hline Age $(\mathrm{y})$ & $29.8(21.9-40.0)$ & $30.0(21.1-39.5)$ & .532 \\
Body height $(\mathrm{cm})$ & $178.4(168.2-185.1)$ & $179.8(170.2-186.9)$ & .906 \\
Body weight $(\mathrm{kg})$ & $73.5(68.5-89.5)$ & $74.4(65.7-88.3)$ & .726 \\
Postsurgery period (mo) & $6.7(3.0-11.0)$ & NA & NA \\
Questionnaire (VISA-A) score for repaired & $74(51-100)$ & & \\
leg $(0-100)^{*}$ & & & \\
\hline NOTE. Values are median (range). & & \\
Abbreviation: NA, not applicable. & \\
$*$ A higher VISA-A score indicates a lower clinical severity.
\end{tabular}

The duration of postsurgical physical therapy ranged from 0 to 12 weeks among the 23 subjects after their supervised 16-week protocol. Six of the 16 control subjects were excluded because they had signs of asymptomatic Achilles' tendinopathy during ultrasonographic screenings; thus there were 10 subjects in the control group ( 5 badminton players, 2 football players, 2 basketball players, 1 volleyball player). There was no significant difference in the physical characteristics of the subjects in either the injured or healthy control groups as shown by the MannWhitney $U$ tests (see table 1 ).

\section{Mechanical and myoelectrical recording}

All measurements were taken for both legs in the order of a block randomization scheme using the techniques described in our previous studies. $^{6,21}$ Each subject lay prone (face down) on an examination bed with both ankles hanging over the edge of the bed. ${ }^{6,21}$ A load cell ${ }^{\mathrm{b}}$ connected to the footplate was used to record voluntary or evoked isometric torque. The foot was fixed on a footplate placed at $90^{\circ}$ to the tibia. Ankle fixation was assessed by continuous recording of the degree of ankle flexion by an electrogoniometer ${ }^{\mathrm{c}}$ with ends fixed to the medial side of the lower leg and to the medial side of the foot. This electrogoniometer was connected to an MP100 system $^{\mathrm{d}}$ (same for the load cell). Myoelectrical activities of the triceps surae and tibialis anterior were measured using 4 pairs of TSD150B active-surface electromyographic recording electrodes. ${ }^{21}$ A portable electromyography instrument ${ }^{\mathrm{e}}$ was used to ensure that the interelectrode resistance was less than $5 \mathrm{k} \Omega$.

\section{Electrical stimulation}

To compare muscle activity among different subjects, the electromyographic amplitudes of voluntary muscle activity were normalized by the electrically evoked amplitude of the $\mathrm{M}$ wave. The posterior tibial nerve was stimulated using an electrical stimulator, ${ }^{f}$ with the cathode (diameter, $0.5 \mathrm{~cm}$ ) and the anode $(10 \times 5 \mathrm{~cm})$ placed at the popliteal fossa and anterior surface (suprapatellar) of the knee, respectively. ${ }^{6,22}$ A series of submaximal electrical stimulations (increments of $2 \mathrm{~mA}$ from $2 \mathrm{~mA}$; duration, 1ms; waveform, rectangular) were applied with intervals of 10 seconds until maximum values of the torque of the evoked contraction and maximal $\mathrm{M}$-wave $\left(\mathrm{M}_{\max }\right)$ amplitudes were obtained. Ten minutes after the above tests, the subjects were instructed to contract their calf muscles "as fast and as forcefully as possible" and maintain their maximal voluntary isometric contraction (MVIC) in plantar flexion for 5 seconds, followed by a 2-minute rest between each trial. A superimposed supramaximal intensity stimulation current $(86-110 \mathrm{~mA})$ was $50 \%$ greater than that applied at $\mathrm{M}_{\max }$, and was applied at the time of the midpoint of peak torque in each trial to record the superimposed supramaximal $\mathrm{M}$ wave $\left(\mathrm{M}_{\text {sup }}\right)$ for the triceps surae..$^{22,23}$

\section{Mechanical properties}

For this measurement, each subject was instructed to gradually increase plantar flexion force in their foot from a relaxed status to MVIC within 5 seconds, maintain MVIC for 1 second, and then gradually relax, achieving complete relaxation in 5 seconds. $^{10,21,24}$ Tendon elongation measurements were taken during the ramping contraction, MVIC, and relaxation by $5-$ to $10-\mathrm{MHz}$ B-mode ultrasonography ${ }^{\mathrm{g}}$ using the techniques described in our previous studies. $^{21}$ For synchronization, software containing simulating switching circuits written using Labview $7.1^{\mathrm{h}}$ was used to add audioelectrical signals to the camera and MP100 system ${ }^{\mathrm{d}}$ at the beginning and end of each measurement. Displacement digitalization of the junction was performed by the same examiner by using MATLAB 7.1 software. ${ }^{i}$ To ascertain the negative forces from the tibialis anterior cocontraction during plantar flexion, each subject also performed 5-second isometric contractions and relaxation of dorsiflexion. The negative forces of the tibialis anterior during plantar flexion were estimated by the cocontraction electromyographic activities and the relationship between the dorsiflexion force and the root mean square (RMS) electromyography. Corrected displacements of the myotendinous junction of the gastrocnemius on the ultrasonographic images, with adjusted corresponding tendon force values during the ascending (contraction) and descending (relaxation) phases, were fitted with second-order polynomial functions. $^{20,24}$ Tendon stiffness was defined as the slope of the ascending phase of muscle contraction between $50 \%$ and $100 \%$ of the maximum force. ${ }^{20,24}$ The calculated ratio of the area within the stress-strain loop to the area beneath the curve during the ascending phase was defined as hysteresis. ${ }^{10,24}$ Area calculations were also made with MATLAB 7.1 software. $^{\mathrm{i}}$

\section{One-leg hopping test}

After 10 minutes of rest, the subjects stood behind a takeoff line marked on the floor. Tests started from an erect standing position, and subjects were instructed to jump on the front of their foot as far as possible for 1-leg jumping, without losing balance or stepping onto the other leg. The subjects were allowed to maximize their forward drive by performing a countermovement of quick knee bending and backward arm swing before the jump. Subjects performed at least 3 maximal trials for each leg, with the longest hopping distance for each leg recorded for analysis. The approved trials were defined as 3 trials with no successive increases. 


\section{Star Excursion Balance Test}

Subjects stood on 1 leg in the center of a grid on the floor, which was formed by 3 lines extending as a "Y." 23 Subjects reached in the anterior, posteromedial, and posterolateral directions as far as possible with the free foot, without losing balance or touching down the reaching (free) foot. ${ }^{25}$ The greatest of the 3 trials of each direction (ie, the most distance between the grid and the point the reaching foot touches) was recorded and normalized by the leg length (anterior superior iliac spine to lateral malleolus). The composite score was calculated according to methods described in previous studies. ${ }^{25}$

\section{Data processing}

\section{Rate of force development}

During offline analysis, the load-cell signal was filtered by a digital fourth-order, zero-lag, recursive Butterworth low-pass filter, with an upper cutoff frequency of $50 \mathrm{~Hz} .{ }^{23}$ Subsequently, the load-cell force signal was multiplied by the horizontal distance between the lateral malleolus and the ball of the foot to calculate the torque. ${ }^{26}$ The absolute RFD was derived as the average slope of the torque-time curve ( $\Delta$ torque/ $\Delta$ time) over time spans of 0 to 100 and 0 to 200 milliseconds relative to the onset of contraction, determined when the torque exceeded $7.5 \mathrm{Nm}{ }^{7}$

\section{Electromyography}

The electromyography signals were digitally high-pass filtered using a fourth-order, zero-lag Butterworth filter (cutoff frequency, 20Hz) and then a moving RMS filter, with a time constant of 50 milliseconds, ${ }^{23}$ using MATLAB 7.1 software. ${ }^{i}$ The onset of electromyography integration was set to 30 milliseconds before the onset of torque (fig 1). ${ }^{6,27}$ The following were the surface electromyography parameters of the triceps surae and tibialis anterior, as defined from the onset of electromyography integration: (1) normalized RER (the slope of $\Delta$ electromyography/ $\Delta$ time), determined for time spans of 0 to 50 and 0 to 75 milliseconds and normalized to the $\mathrm{M}_{\text {sup }}$; and (2) the RER ratio between the tibialis anterior and soleus in the time spans of 0 to 50 and 0 to 75 milliseconds. ${ }^{6}$

\section{Statistical analyses}

Legs were divided into 3 groups according to the injury history or subject: group 1, legs with Achilles' tendon repairs; group 2, noninjured contralateral legs in athletes with an Achilles' tendon repair; and group 3, legs of the healthy control subjects. Since there were no significant differences between the 2 legs in the control group, data for both sides were averaged to represent the result of the outcome. All data were averaged over at least 3 trials. All data are presented as medians (range from minimum to maximum). Kruskal-Wallis 1-way analysis of variance by ranks was performed to test whether samples in each of the 3 different groups originated from the same distribution. Post hoc procedures with a modified alpha level of $P<.016$ were used to determine the significance between the Achilles' tendon repair group and each control group. ${ }^{28}$ Correlations between the neuromechanical variables and performances were assessed using Spearman correlation tests. Data were analyzed using SPSS 16.0 software $^{\mathrm{j}}$ with the alpha level set at .05 .

\section{Results}

Compared with the noninjured contralateral legs or the legs of the healthy controls, the repaired legs of the athletes showed lower normalized RERs in the soleus, gastrocnemius medialis, and gastrocnemius lateralis muscles at 0 to 50 milliseconds and at 0 to 75 milliseconds (table 2), less tendon stiffness, greater mechanical hysteresis during ramping isometric contraction, less RFD at 0 to

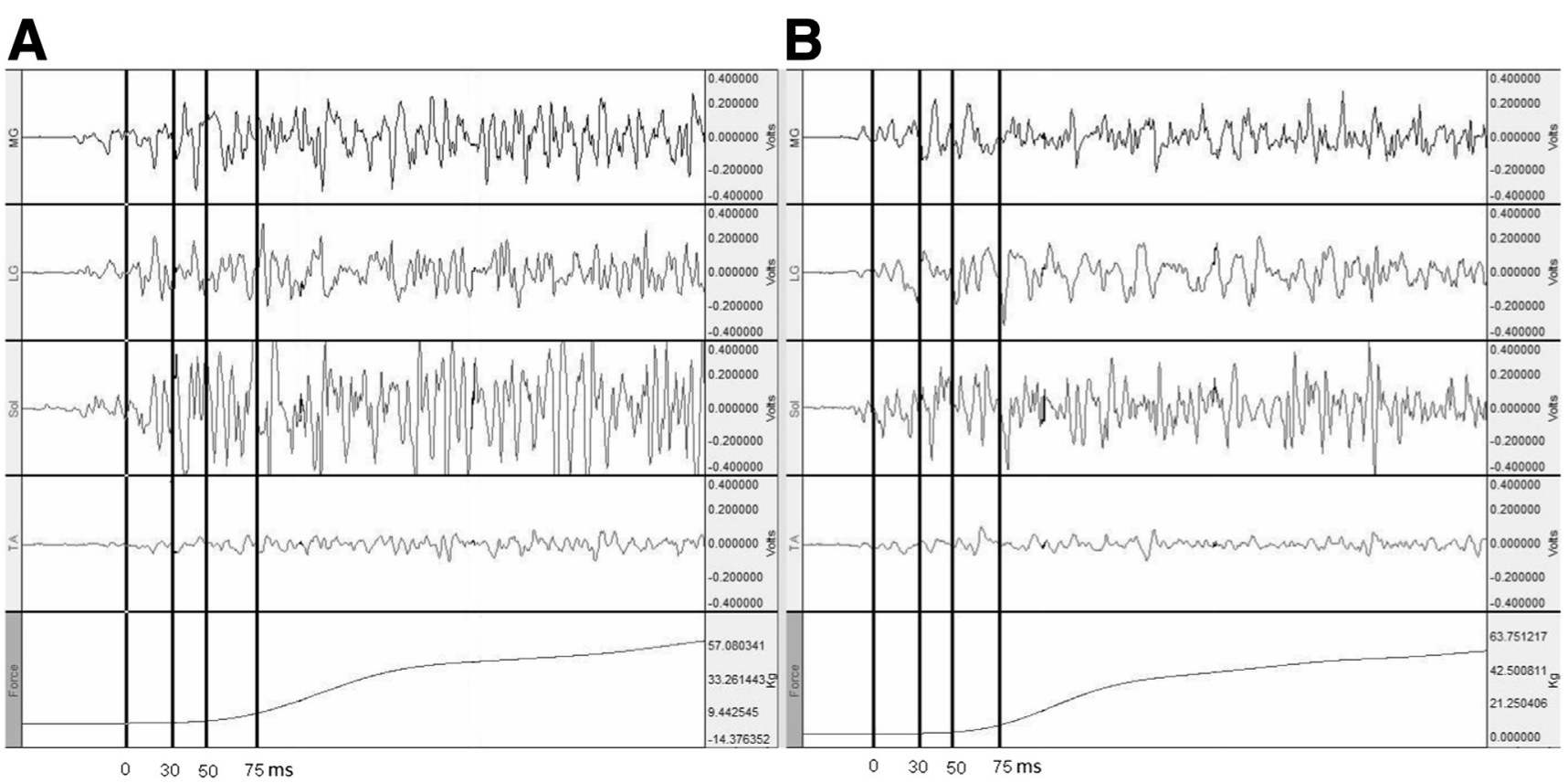

Fig 1 Example of electromyography of triceps surae muscles and voluntary force recorded from the initiation of a maximal voluntary plantar flexion contraction for 1 subject. (A) Noninjured contralateral leg. (B) Leg with repaired Achilles' tendon. Vertical lines indicate 0, 30, 50, and 75 milliseconds relative to electromyography onset. Abbreviations: LG, gastrocnemius lateralis; MG, gastrocnemius medialis; SOL, soleus; TA, tibialis anterior. 
Table 2 Kruskal-Wallis 1-way analysis of neuromechanical outcomes and explosive performances in 3 different leg groups

\begin{tabular}{|c|c|c|c|c|}
\hline \multirow[b]{2}{*}{ Variable and Unit } & \multirow[b]{2}{*}{ Repaired Leg } & \multirow[b]{2}{*}{ Noninjured Leg } & \multirow[b]{2}{*}{ Control Leg } & \multirow{2}{*}{$\begin{array}{l}\text { Kruskal-Wallis } \\
\text { Significant Comparison and Post Hoc }(P<.016)\end{array}$} \\
\hline & & & & \\
\hline NorRER $(0-50 \mathrm{~ms}) \mathrm{SOL}(\mathrm{mV} / \mathrm{s}) / \mathrm{mV}$ & $0.33(0.15-0.82)$ & $0.42(0.25-0.89)$ & $0.60(0.48-0.75)$ & $\begin{array}{l}\text { Repaired and control legs }(P=.001) \\
\text { Noninjured and control legs }(P=.011)\end{array}$ \\
\hline NorRER $(0-50 \mathrm{~ms}) \mathrm{MG}(\mathrm{mV} / \mathrm{s}) / \mathrm{mV}$ & $0.64(0.13-1.21)$ & $0.67(0.41-1.33)$ & $0.79(0.68-0.95)$ & NS (all $P>.016$ ) \\
\hline NorRER $(0-50 \mathrm{~ms})$ LG $(\mathrm{mV} / \mathrm{s}) / \mathrm{mV}$ & $0.49(0.28-1.06)$ & $0.64(0.35-1.10)$ & $0.82(0.61-1.10)$ & $\begin{array}{l}\text { Repaired and control legs }(P=.004) \\
\text { Noninjured and control legs }(P=.011)\end{array}$ \\
\hline NorRER $(0-75 \mathrm{~ms})$ SOL $(\mathrm{mV} / \mathrm{s}) / \mathrm{mV}$ & $0.17(0.07-0.54)$ & $0.24(0.11-0.61)$ & $0.29(0.20-0.41)$ & $\begin{array}{l}\text { Repaired and noninjured legs }(P=.004) \\
\text { Repaired and control legs }(P=.006)\end{array}$ \\
\hline NorRER $(0-75 \mathrm{~ms}) \mathrm{MG}(\mathrm{mV} / \mathrm{s}) / \mathrm{mV}$ & $0.28(0.07-0.72)$ & $0.45(0.22-0.79)$ & $0.57(0.26-0.74)$ & $\begin{array}{l}\text { Repaired and noninjured legs }(P=.001) \\
\text { Repaired and control legs }(P=.004)\end{array}$ \\
\hline NorRER $(0-75 \mathrm{~ms})$ LG $(\mathrm{mV} / \mathrm{s}) / \mathrm{mV}$ & $0.28(0.13-0.61)$ & $0.37(0.14-0.65)$ & $0.41(0.28-0.56)$ & Repaired and noninjured legs $(P=.004)$ \\
\hline $\operatorname{RER}(0-50 \mathrm{~ms})$ ratio $(\mathrm{mV} / \mathrm{s}) /(\mathrm{mV} / \mathrm{s})$ & $13.8(6.3-32.8)$ & $16.0(6.7-40.0)$ & $16.4(7.5-30.4)$ & NS (all $P>.016$ ) \\
\hline RER $(0-75 \mathrm{~ms})$ ratio $(\mathrm{mV} / \mathrm{s}) /(\mathrm{mV} / \mathrm{s})$ & $12.5(5.5-31.4)$ & $15.9(5.9-55.7)$ & $20.8(8.1-27.9)$ & NS (all $P>.016$ ) \\
\hline Tendon stiffness ( $\mathrm{N} / \mathrm{mm})$ & $78.1(50.0-156.0)$ & $124.6(98.9-186.6)$ & $131.7(120.6-167.8)$ & $\begin{array}{l}\text { Repaired and noninjured legs }(P<.001) \\
\text { Repaired and control legs }(P<.001)\end{array}$ \\
\hline Mechanical hysteresis (\%) & $0.30(0.23-0.42)$ & $0.23(0.04-0.34)$ & $0.22(0.18-0.31)$ & $\begin{array}{l}\text { Repaired and noninjured legs }(P<.001) \\
\text { Repaired and control legs }(P=.001)\end{array}$ \\
\hline RFD 0-100ms (Nm/s) & $552.8(401.2-1044.0)$ & $711.3(341.1-1158.6)$ & $744.9(666.1-910.2)$ & $\begin{array}{l}\text { Repaired and noninjured legs }(P=.006) \\
\text { Repaired and control legs }(P=.001)\end{array}$ \\
\hline RFD 0-200ms (Nm/s) & $317.8(127.2-530.9)$ & $388.9(166.8-606.6)$ & $387.3(166.8-606.6)$ & Repaired and noninjured legs $(P=.001)$ \\
\hline Torque of MVIC (Nm) & $107.7(76.5-153.8)$ & $114.1(84.3-156.1)$ & $124.3(96.7-146.6)$ & NS (all $P>.016$ ) \\
\hline Ankle dorsiflexion (deg) & $20(13-27)$ & $25(18-32)$ & $26(23-32)$ & $\begin{array}{l}\text { Repaired and noninjured legs }(P<.001) \\
\text { Repaired and control legs }(P<.001)\end{array}$ \\
\hline One-leg hopping distance (m) & $1.70(1.36-1.95)$ & $1.77(1.40-2.13)$ & $1.81(1.40-2.13)$ & Repaired and noninjured legs $(P=.001)$ \\
\hline SEBT $(\%)$ & $84.1(59.3-98.9)$ & $99.0(79.8-122.5)$ & $106.6(82.7-122.5)$ & $\begin{array}{l}\text { Repaired and noninjured legs }(P<.001) \\
\text { Repaired and control legs }(P<.001)\end{array}$ \\
\hline
\end{tabular}

NOTE. Values are median (range) or as otherwise indicated.

Abbreviations: LG, gastrocnemius lateralis; MG, gastrocnemius medialis; NorRER, normalized rate of electromyographic rise; NS, not significant; SOL, soleus. 
100 and 0 to 200 milliseconds during explosive plantar flexion, less joint range of ankle dorsiflexion, a shorter 1-leg hopping distance, and a lower Star Excursion Balance Test (SEBT) composite score (see table 2). The contralateral noninjured legs of the athletes showed a lower normalized RER at 0 to 50 milliseconds in the soleus and lateral gastrocnemius postsurgically than the control legs of the healthy subjects (see table 2) (all $P<.016$ ). The neuromechanical outcomes and performance showed correlations for RFD of plantar flexion, 1-leg hopping distance, and SEBT score $(\rho=-.486$ to $.520, P=.039$ to $<.001)$ (table 3$)$.

\section{Discussion}

This study, which involved athletes who underwent unilateral Achilles' tendon repair surgery in the previous year, showed diverse early deficits in the neuromechanics of the triceps surae muscletendon unit, including activation strategies (RER) during the early stage of explosive contractions and tendon mechanical properties in the repaired legs, when compared with the noninjured contralateral legs or the legs of the healthy controls. Differences in the activation strategies were also found when the uninjured contralateral legs were compared with the control legs. We observed correlations between the neuromechanical outcomes and lower extremity explosive performances in these athletes. These findings in comparison with the healthy control group confirm our hypothesis that altered neuromechanical profiles exist in the legs of athletes within 1 year of surgical Achilles' tendon repair. Although this is not a longitudinal study, these novel findings provide important information regarding clinical practices and conservative treatment of athletes after Achilles' tendon repair.

Our comparison between the different leg groups showed that the athletes recruited $\geq 3$ and $<12$ months postsurgically demonstrated lower normalized RERs in the soleus, gastrocnemius medialis, and gastrocnemius lateralis at 0 to 50 and 0 to 75 milliseconds in the repaired leg (see table 2 ). The noninjured contralateral legs also showed lower normalized RERs at 0 to 50 milliseconds in the soleus and gastrocnemius lateralis muscles when compared with the legs of the healthy controls. The RER represents the ability of the neural system to rapidly activate limb muscles and is a determinant of RFD. ${ }^{7}$ These findings among athletes who had Achilles' tendon repair surgery $<12$ months previously indicate that there is less bilateral ability to rapidly activate agonist muscles in the early stage of explosive contractions or torque development. These bilateral deficits may be caused by postoperative immobilization, restrictions, or limitations to foot/ankle activities within the first 3 months

Table 3 Correlations ( $\rho$ and $P$ ) between neuromechanical outcomes and explosive performance

\begin{tabular}{|c|c|c|c|c|}
\hline \multirow[b]{2}{*}{ Variable and Unit } & \multicolumn{4}{|c|}{ Explosive Performance } \\
\hline & RFD $0-100 \mathrm{~ms}$ & RFD $0-200 \mathrm{~ms}$ & Hopping Distance & SEBT \\
\hline \multicolumn{5}{|c|}{ NorRER $(0-50 \mathrm{~ms}) \mathrm{SOL}(\mathrm{mV} / \mathrm{s}) / \mathrm{mV}$} \\
\hline$\rho=$ & .159 & .023 & .179 & .382 \\
\hline$P=$ & .241 & .865 & .197 & $.004^{*}$ \\
\hline \multicolumn{5}{|c|}{ NorRER $(0-50 \mathrm{~ms}) \mathrm{MG}(\mathrm{mV} / \mathrm{s}) / \mathrm{mV}$} \\
\hline$\rho=$ & .118 & .03 & .293 & .366 \\
\hline$P=$ & .385 & .826 & $.028^{*}$ & $.006^{*}$ \\
\hline \multicolumn{5}{|c|}{ NorRER $(0-50 \mathrm{~ms}) \mathrm{LG}(\mathrm{mV} / \mathrm{s}) / \mathrm{mV}$} \\
\hline$\rho=$ & .351 & .150 & .133 & .363 \\
\hline$P=$ & $.008^{*}$ & .270 & .329 & $.006^{*}$ \\
\hline \multicolumn{5}{|c|}{ NorRER $(0-75 \mathrm{~ms})$ SOL $(\mathrm{mV} / \mathrm{s}) / \mathrm{mV}$} \\
\hline$\rho=$ & .104 & .369 & .240 & .403 \\
\hline$P=$ & .447 & $.005^{*}$ & .075 & $.002^{*}$ \\
\hline \multicolumn{5}{|c|}{ NorRER $(0-75 \mathrm{~ms}) \mathrm{MG}(\mathrm{mV} / \mathrm{s}) / \mathrm{mV}$} \\
\hline$\rho=$ & .389 & .520 & .510 & .512 \\
\hline$P=$ & $.003^{*}$ & $<.001^{*}$ & $<.001^{*}$ & $<.001^{*}$ \\
\hline \multicolumn{5}{|c|}{ NorRER $(0-75 \mathrm{~ms}) \mathrm{LG}(\mathrm{mV} / \mathrm{s}) / \mathrm{mV}$} \\
\hline$\rho=$ & .277 & .413 & .164 & .316 \\
\hline$P=$ & $.039^{*}$ & $.002^{*}$ & .228 & $.018^{*}$ \\
\hline \multicolumn{5}{|c|}{ RER $(0-50 \mathrm{~ms})$ ratio $(\mathrm{mV} / \mathrm{s}) /(\mathrm{mV} / \mathrm{s})$} \\
\hline$\rho=$ & -.027 & .024 & .293 & .300 \\
\hline$P=$ & .846 & .859 & .059 & $.025^{*}$ \\
\hline \multicolumn{5}{|c|}{$\operatorname{RER}(0-75 \mathrm{~ms})$ ratio $(\mathrm{mV} / \mathrm{s}) /(\mathrm{mV} / \mathrm{s})$} \\
\hline$\rho=$ & -.085 & -.121 & .254 & .440 \\
\hline$P=$ & .534 & .374 & .059 & $<.001^{*}$ \\
\hline \multicolumn{5}{|c|}{ Tendon stiffness $(\mathrm{N} / \mathrm{mm})$} \\
\hline$\rho=$ & .388 & .328 & .427 & .546 \\
\hline$P=$ & $.003^{*}$ & $.014^{*}$ & $.001^{*}$ & $<.001^{*}$ \\
\hline \multicolumn{5}{|c|}{ Mechanical hysteresis (\%) } \\
\hline$\rho=$ & -.292 & -.372 & -.423 & -.486 \\
\hline$P=$ & $.029^{*}$ & $.005^{*}$ & $.001^{*}$ & $<.001^{*}$ \\
\hline
\end{tabular}

Abbreviations: LG, gastrocnemius lateralis; MG, gastrocnemius medialis; NorRER, normalized rate of electromyographic rise; SOL, soleus.

* Correlations are significant. 
after Achilles' tendon repair. These findings strongly suggest that there is a need to extend the common 16-week protocol for athletes into the third month postsurgery to minimize these bilateral neural deficits. ${ }^{22,29}$ In addition, care must be taken in interpreting results when using the noninjured leg as the reference for postsurgical rehabilitation because reduced explosive muscle activation in the noninjured leg may lead to invalid muscle tests.

Lower tendon stiffness in the repaired legs of athletes $\geq 3$ and $<12$ months postsurgery was observed, compared with contralateral and control legs (see table 2). These results are consistent with the magnetic resonance imaging-based findings of Schepull et al, ${ }^{3}$ who observed stiffness increased from $63 \mathrm{~N} / \mathrm{mm}$ at 18 weeks to $134 \mathrm{~N} / \mathrm{mm}$ at 1 year postsurgery in recreational athletes. Our findings indicate incomplete tendon healing, in terms of tendon structure and composition, until 12 months after surgery. Reduced stiffness in the repaired tendon would impede quick movement production by requiring additional time to stretch the series elastic component in the myotendinous complex and thus lead to a lower RFD, as found in this study. This low level of stiffness also constrains the neuromuscular ability to (1) achieve accurate postural stability or positioning of a limb segment ${ }^{11}$; and (2) achieve a given position after a perturbation. ${ }^{30}$ Tendon stiffness is associated with muscle strength and can be enhanced by plyometric or resistance training. ${ }^{21,31}$ We recommend a progressive resistance training from the third month after repair for an earlier recovery of tendon mechanical strength, a step that has been omitted in previous studies. ${ }^{32}$ This training, under supervision, starts at high repetition/ light resistance and proceeds to low repetition/high resistance to build tendon strength gradually. Compared with the noninjured and control legs, both lower tendon stiffness and greater tendon mechanical hysteresis were found in the repaired legs. Increased mechanical hysteresis in the repaired leg indicates reduced energyconserving capacity and increased energy dissipation as heat in repaired legs resulting from tendon internal viscous damping and friction between the tendon and paratendon. ${ }^{10}$ Since deep friction massages may loosen the adhesion between the tendon and peritendinous tissues, it is suggested that further studies investigate the effects of deep friction massages or tendon mobilization procedures in reducing the hysteresis that occurs after Achilles' tendon repairs.

Comparisons of explosive performances between the 3 leg groups showed that, for the legs $\geq 3$ and $<12$ months after Achilles' tendon repair, the RFD was lower, ankle dorsiflexion was less, the 1-leg hopping distance was shorter, and lower extremity stability (SEBT composite score) was less when compared with the contralateral legs of athletes after surgery or with the legs of healthy controls (see table 2). The decreased ability to develop force rapidly is suggested to be associated with a lower capacity for neuromuscular response in controlling postural sway. ${ }^{33}$ Balance exercises for the repaired legs of athletes, as well as various exercises of an explosive nature, are strongly recommended to prevent functional deficits in balance after Achilles' tendon repairs. Reduced ankle dorsiflexion was also found in the repaired leg. This could lead to overload of the triceps surae muscle-tendon units during landing impact. $^{34}$ Therefore, we also recommend stretching exercises to improve dorsiflexion flexibility early after the 16 -week protocol. ${ }^{12,13}$

Two characteristics among correlations between explosive performance of the lower extremities and the above-mentioned neuromechanical profiles were found (see table 3). First, there were correlations between agonist activation and antagonist coactivation (normalized RER and RER ratios) during early-stage fast contractions and explosive or balance performance, such as RFD, hopping distance, and SEBT scores. Our finding is consistent with previous studies that suggest that RER is a determinant of RFD. ${ }^{7}$ In addition, our results indicate that fast neural activation and coordination between agonist and antagonist muscles determine fast force capacity and hopping distance, and also affect balance ability. Second, tendon mechanical properties such as stiffness and mechanical hysteresis were associated with explosive performance, including RFD, hopping distance, and SEBT scoring. These results are consistent with those of previous studies $^{11,31,35}$ and also indicate that the mechanical properties of the tendon-aponeurosis complex after surgery affect RFD, jump performance, and balance capacity. We recommend treatments such as combination strength and power training from low resistance $^{2}$ beginning 3 months after Achilles' tendon repairs, to improve fast activation, reduce coactivation in the leg muscle, and enhance stiffness.

Based on the findings of this study, a 6-month physical therapy period is suggested to prevent bilateral neuromechanical deficits in the triceps surae muscle-tendon unit in athletes after Achilles' tendon repair. This 6-month program lasts longer than current treatment guidelines because there is additional neuromechanical training. The part of the program regarding progressive resistance, stretching, and coordination training has not been addressed in previous studies. This 6-month program starts with the common 16-week protocol ${ }^{12,13}$ or early functional rehabilitation, ${ }^{36,37}$ and integrates progressive resistance, stretching, and balance exercises after the repairs. Isometric resistance exercises can be combined with electrotherapy and deep friction massage starting 3 months after surgery in the repaired legs to minimize the neural deficits and tendon mechanical hysteresis. Progressive resistance exercises and stretching can be started in the fourth month after surgery and include progressive weight-bearing and stretch training to increase activation, tendon stiffness, and flexibility. Bilateral power training and full-range stretching exercises are performed for coordination in the fifth month after repair.

There are 2 current postoperative rehabilitation protocols after repair of an Achilles' tendon rupture: the 16-week protocol and the early functional rehabilitation protocol. ${ }^{12,13,36,37}$ The 16 -week protocol commonly includes a 6-week cast immobilization, which is then followed by functional rehabilitation for another 10 weeks. ${ }^{12,13}$ The early functional treatment protocol involves weight-bearing that would start as early as the first postoperative day. In addition, this early protocol includes using an early range of motion and splinting in a modified orthosis with a locking hinge from the first to the sixth week. This is then followed by a supervised, graduated strength program during weeks 6 through 12 after surgery, with the aim of returning the athlete to a preinjury level of sporting activity by 4.5 to 6 months. ${ }^{36,37}$ Although both protocols are well accepted in clinics, neither of them introduces a detailed program for rehabilitation starting from the third month after the repair. Our suggestion-based on the results of this study-is to provide supplemental exercise prescriptions to both protocols. In addition, these prescriptions may be feasibly used in injured athletes receiving nonoperative treatment of Achilles' tendon rupture. Future studies are recommended to investigate whether an integrated, early rehabilitation protocol would have eliminated or minimized the observed deficits in this cohort study.

\section{Study limitations}

The limitations of this study include its retrospective study design, small sample size, and the specific population that was studied. As 
well, there was a large range $(0-12 w k)$ with regard to the time of postsurgical rehabilitation. This perhaps impacted our data analyses because injured athletes with different rehabilitation histories were all classified together. In addition, muscle activation appears to depend on the task type, ${ }^{38}$ and an isometric test environment may not provide optimal conditions to observe adaptations in all muscles. Nevertheless, the muscle activations of the gastrocnemius muscles included in this study have not been investigated in previous studies. $^{2}$ Our results showed postsurgical neuromechanical alterations in 3 calf muscles, leading to deficits in performances with regard to explosive power.

\section{Conclusions}

Bilateral alterations of neural activation strategies and tendon mechanical properties are found in the triceps surae muscletendon units in athletes within 1 year of unilateral Achilles' tendon repair. Neuromechanical profiles of the triceps surae muscle-tendon unit correlated with lower extremity explosive performances.

\section{Suppliers}

a. EnVisor; Philips Medical Systems Inc, 2301 5th Ave \#200, Seattle, WA 98121.

b. SLS410-Load Cell; Mettler-Toledo Pac Rim AG, 2F, No. 17 Lane 171, Sec. 2, Jiu Zong Rd, Taipei 114, Taiwan.

c. Sharp Sensor S700; Measurand Inc, 2111 Hanwell Rd, Fredericton, NB, Canada E3C 1M7.

d. Biopac, 42 Aero Camino, Santa Barbara, CA 93117.

e. Sierra II; Cadwell Laboratories Inc, 909 N Kellogg St, Kennewick, WA 99336.

f. Digitimer Stimulator DS7AH; Digitimer Ltd, 37 Hydeway, Welwyn Garden City, Hertfordshire, AL7 3BE UK.

g. SonoSite Inc, 21919 30th Drive SE, Bothell, WA 98021.

h. National Instruments, $11500 \mathrm{~N}$ Mopac Expwy, Austin, TX 78759-3504.

i. MathWorks, 3 Apple Hill Dr, Natick, MA 01760-2098.

j. SPSS Inc, 233 S Wacker Dr, 11th Fl, Chicago, IL 60606.

\section{Keywords}

Mechanical phenomena; Nervous system; Postural balance; Rehabilitation; Tendons

\section{Corresponding author}

Hsing-Kuo Wang, PT, MSc, MMedSci, PhD, Sports Physiotherapy Laboratory, School and Graduate Institute of Physical Therapy, College of Medicine, National Taiwan University, Floor 3, No. 17, Xuzhou Rd, Zhongzheng District, Taipei City 100, Taiwan (ROC). E-mail address: hkwang@ntu.edu.tw.

\section{References}

1. Leppilahti J, Puranen J, Orava S. Incidence of Achilles tendon rupture. Acta Orthop Scand 1996;67:277-9.

2. Don R, Ranavolo A, Cacchio A, et al. Relationship between recovery of calf-muscle biomechanical properties and gait pattern following surgery for Achilles tendon rupture. Clin Biomech 2007; 22:211-20.

3. Schepull T, Kvist J, Andersson C, Aspenberg P. Mechanical properties during healing of Achilles tendon ruptures to predict final outcome: a pilot Roentgen stereophotogrammetric analysis in 10 patients. BMC Musculoskelet Disord 2007;8:116.

4. Basmajian JV. Motor learning and control: a working hypothesis. Arch Phys Med Rehabil 1977;58:38-41.

5. Hagood S, Solomonow M, Baratta R, Zhou BH, D'Ambrosia R. The effect of joint velocity on the contribution of the antagonist musculature to knee stiffness and laxity. Am J Sports Med 1990;18:182-7.

6. Wang HK, Lin KH, Wu YK, Chi SC, Shih TT, Huang YC. Evoked spinal reflexes and force development in elite athletes with middleportion Achilles tendinopathy. J Orthop Sports Phys Ther 2011;41: 785-94.

7. Aagaard P, Simonsen EB, Andersen JL, Magnusson P, DyhrePoulsen P. Increased rate of force development and neural drive of human skeletal muscle following resistance training. J Appl Physiol 2002;93:1318-26.

8. Magnusson SP, Aagaard P, Dyhre-Poulsen P, Kjaer M. Loaddisplacement properties of the human triceps surae aponeurosis in vivo. J Physiol 2001;531:277-88.

9. Magnusson SP, Narici MV, Maganaris CN, Kjaer M. Human tendon behaviour and adaptation, in vivo. J Physiol 2008;586:71-81.

10. Maganaris CN, Paul JP. Hysteresis measurements in intact human tendon. J Biomech 2000;33:1723-7.

11. Onambele GL, Narici MV, Maganaris CN. Calf muscle-tendon properties and postural balance in old age. J Appl Physiol 2006;100:2048-56.

12. Strom AC, Casillas MM. Achilles tendon rehabilitation. Foot Ankle Clin 2009; 14:773-82.

13. Bostick GP, Jomha NM, Suchak AA, Beaupré LA. Factors associated with calf muscle endurance recovery 1 year after Achilles tendon rupture repair. J Orthop Sports Phys Ther 2010;40:345-51.

14. Bryant AL, Clark RA, Bartold S, et al. Effects of estrogen on the mechanical behavior of the human Achilles tendon in vivo. J Appl Physiol 2008;105:1035-43.

15. Maffulli N, Kenward MG, Testa V, Capasso G, Regine R, King J. Clinical diagnosis of Achilles tendinopathy with tendinosis. Clin J Sport Med 2003;13:11-5.

16. O'Reilly MA, Massouh H. Pictorial review: the sonographic diagnosis of pathology in the Achilles tendon. Clin Radiol 1993;48:202-6.

17. Sofka CM, Adler RS, Positano R, Pavlov H, Luchs JS. Haglund's syndrome: diagnosis and treatment using sonography. HSS J 2006;2: 27-9.

18. Robinson JM, Cook JL, Purdam C, et al. The VISA-A questionnaire: a valid and reliable index of the clinical severity of Achilles tendinopathy. Br J Sports Med 2001;35:335-41.

19. Graven-Nielsen T, Arendt-Nielsen L. Impact of clinical and experimental pain on muscle strength and activity. Curr Rheumatol Rep 2008;10:475-81.

20. Alfredson H. Chronic midportion Achilles tendinopathy: an update on research and treatment. Clin Sports Med 2003;22:727-41.

21. Wu YK, Lien YH, Lin KH, Shih TT, Wang TG, Wang HK. Relationships between three potentiation effects of plyometric training and performance. Scand J Med Sci Sports 2010;20:e80-6.

22. Gondin J, Duclay J, Martin A. Soleus- and gastrocnemii-evoked $\mathrm{V}$ wave responses increase after neuromuscular electrical stimulation training. J Neurophysiol 2006;95:3328-35.

23. Un CP, Lin KH, Shiang TY, Chang EC, Su SC, Wang HK. Comparative and reliability studies of neuromechanical leg muscle performances of volleyball athletes in different divisions. Eur J Appl Physiol 2013;113:457-66.

24. Wang HK, Lin KH, Su SC, Shih TT, Huang YC. Effects of tendon viscoelasticity in Achilles tendinosis on explosive performance and clinical severity in athletes. Scand J Med Sci Sports 2012;22:e147-55.

25. Filipa A, Byrnes R, Paterno MV, Myer GD, Hewett TE. Neuromuscular training improves performance on the star excursion balance test in young female athletes. J Orthop Sports Phys Ther 2010;40:551-8. 
26. Sasaki K, Ishii N. Shortening velocity of human triceps surae muscle measured with the slack test in vivo. J Physiol 2005;567:1047-56.

27. Jubeau M, Zory R, Gondin J, Martin A, Maffiuletti NA. Late neural adaptations to electrostimulation resistance training of the plantar flexor muscles. Eur J Appl Physiol 2006;98:202-11.

28. Conover WJ. Practical nonparametric statistics. 3rd ed. New York: Wiley; 1999. p 288-93.

29. Maffiuletti NA, Pensini M, Martin A. Activation of human plantar flexor muscles increases after electromyostimulation training. J Appl Physiol 2002;92:1383-92.

30. Biewener AA, Roberts TJ. Muscle and tendon contributions to force, work and elastic energy savings: a comparative perspective. Exerc Sport Sci Rev 2000;28:99-107.

31. Muraoka T, Muramatsu T, Fukunaga T, Kanehisa H. Elastic properties of human Achilles tendon are correlated to muscle strength. J Appl Physiol 2005;99:665-9.

32. Maffulli N, Tallon C, Wong J, Lim KP, Bleakney R. Early weightbearing and ankle mobilization after open repair of acute midsubstance tears of the Achilles tendon. Am J Sports Med 2003;1: 692-700.
33. Izquierdo $\mathrm{M}$, Aguado $\mathrm{X}$, Gonzalez R, López JL, Häkkinen $\mathrm{K}$. Maximal and explosive force production capacity and balance performance in men of different ages. Eur J Appl Physiol Occup Physiol 1999;79:260-7.

34. Whitting JW, Steele JR, McGhee DE, Munro BJ. Dorsiflexion capacity affects Achilles tendon loading during drop landings. Med Sci Sports Exerc 2011;43:706-13.

35. Wilson GJ, Murphy AJ, Pryor JF. Musculotendinous stiffness: its relationship to eccentric, isometric, and concentric performance. J Appl Physiol 1994;76:2714-9.

36. Suchak AA, Spooner C, Reid DC, Jomha NM. Postoperative rehabilitation protocols for Achilles tendon ruptures: a meta-analysis. Clin Orthop Relat Res 2006;445:216-21.

37. Willits K, Amendola A, Bryant D, et al. Operative versus nonoperative treatment of acute Achilles tendon ruptures: a multicenter randomized trial using accelerated functional rehabilitation. J Bone Joint Surg Am 2010;92:2767-75.

38. Doorenbosch CA, van Ingen Schenau GJ. The role of mono- and biarticular muscles during contact control leg tasks in man. Hum Mov Sci 1995;14:279-300. 